\title{
Aquaporins in the brain: from aqueduct to "multi-duct"
}

\author{
Jérôme Badaut • Jean-François Brunet • Luca Regli
}

Published online: 15 August 2007

(C) Springer Science + Business Media, LLC 2007

\begin{abstract}
The aquaporin channel family was first considered as a family of water channels, however it is now clear that some of these channels are also permeable to small solutes such glycerol, urea and monocarboxylates. In this review, we will consider AQP4 and AQP9 expressed in the rodent brain. AQP4 is present on astrocytic end-feet in contact with brain vessels and could be involved in ionic homeostasis. However, AQP4 may also be involved in cell adhesion. AQP4 expression is highly modified in several brain disorders and it can play a key role in the cerebral edema formation. However, the exact role of AQP4 in edema formation is still debated. Recently, AQP4 has been shown to be also involved in astrocyte migration during glial scar formation. AQP9 is expressed in astrocytes and in catecholaminergic neurons. Two isoforms of AQP9 are expressed in brain cells, the shortest isoform is localized in the inner membrane of mitochondria and the longest in the cell membrane. The level of expression of AQP9 is negatively regulated by high concentrations of insulin. Taken together, these results suggest that AQP9 could be involved in brain energy metabolism. The induction of AQP9 in astrocytes is observed with time after stroke onset suggesting participation in the clearance of excess lactate in the extracellular space. These recent exciting results suggest that AQPs may not only be involved in water homeostasis in the brain but could also participate in other important physiological functions.
\end{abstract}

Keywords Water channel - Glycerol - Lactate Catecholaminergic neurons ·

Edema $\cdot$ Ischemia

Water is an important molecule involved in several biochemical processes present in living cells. Water was considered for a long time to freely diffuse through the plasma membrane, but this hypothesis was revisited after the discovery of water

J. Badaut $(\bowtie) \cdot$ J.-F. Brunet $\cdot$ L. Regli

Department of Neurosurgery, CHUV-UNIL, 1011 Lausanne, Switzerland

e-mail: jerome.badaut@chuv.ch 
channels (Preston et al. 1992). The water channel family is still growing with 13 members ubiquitously distributed in mammalian tissues. The importance of these channels for life is supported by the fact that there are more than 150 types of AQPs in microbes, invertebrates, non mammalian vertebrates and plants (Santoni et al. 2000).

These channels have been highly conserved throughout evolution and the family is now divided accordingly to sequence homology and permeability into aquaporins and aquaglyceroporins.

Research in this field has significantly advanced in the last years, and in this review we will illustrate several new potential functional roles for brain AQPs.

\section{Water channels: a general introduction}

Aquaporins (AQP) are water channel proteins with a molecular weight of around $30 \mathrm{kDa}$, and exhibit a common structure of six membrane spanning alpha helical domains with intracellular carboxyl $(\mathrm{C})$ and amino $(\mathrm{N})$ termini. They contain a consensus motif Asn-Pro-Ala (NPA), implied in pore formation (Badaut et al. 2002). As mentioned above, the AQP family is now divided into two subgroups based on sequence homology (Amiry-Moghaddam and Ottersen 2003): aquaporin and aquaglyceroporin.

The subgroup aquaporin is composed of AQP0, 1, 2, 4, 5, 6, 8 and is considered to be mainly permeable to water with a high flow rate. A few of these pure water channels are also permeable to anion (AQP6) and volatile solutes such as $\mathrm{CO}_{2}$ for AQP1 (Cooper et al. 2002). Water diffusion through AQPs is inhibited by mercury, except AQP4 which is a mercury-insensitive aquaporin (Amiry-Moghaddam and Ottersen 2003).

The second subgroup aquaglyceroporins, is composed of AQP3, 7, 9, 10 and bacterial glycerol facilitator (Glpf; Badaut and Regli 2004). These channels are permeable to water and glycerol. AQP9, a member of this group, was also surnamed "neutral channel" (Tsukaguchi et al. 1998). Indeed, the presence of AQP9 in Xenopus Oocytes or proteoliposomes injected facilitates the diffusion of water, but also polyols (glycerol, mannitol, and sorbitol), purines (adenine), pyrimidines (uracil and chemotherapeutic agent 5-fluorouracil) and monocarboxylates (lactate and $\beta$ hydroxybutyrate; Ishibashi et al. 1998; Tsukaguchi et al. 1998; Ko et al. 1999; Tsukaguchi et al. 1999; Carbrey et al. 2003). However, the osmotic water coefficient for AQP9 is lower than in a pure water channel like AQP4 (Carbrey et al. 2003). In addition, AQP9 facilitates metalloid transport further suggesting that APQ9 may be a major route of arsenite uptake into mammalian cells (Liu et al. 2002).

Water homeostasis is critical to sustain normal neural activity. An increase in the water content into the brain leads to brain swelling and rapidly becomes deleterious. Obviously, the knowledge of the distribution and regulation of water channels in the brain is important to understand water homeostasis. To date, six aquaporin subtypes (AQP1, AQP3, AQP4, AQP5, AQP8, AQP9 have been described in rodent brain cells. However, only three aquaporins have been clearly identified in brain cells in vivo: AQP1, AQP4 and AQP9 (Badaut et al. 2002). AQP1 is expressed in epithelial cells of the choroid plexus (Nielsen et al. 1993), and is proposed to be involved in cerebrospinal fluid formation (Brown et al. 2004). Expression of AQP1 is seen in 
many non-brain endothelia (Nielsen et al. 1993) but its expression is suppressed in the specialized endothelial cells of the blood-brain barrier (Dolman et al. 2005). Indeed, the presence of astrocytes inhibits the expression of AQP1 in endothelial cells (Dolman et al. 2005). In this review, we will focus on distributions and putative roles of AQP4 and 9 in the mammalian brain. Recent reports concerning the level of expression of AQP in brain disorders will be then presented.

\section{AQP4 expression in brain and its several functional roles}

Historically, mRNA for AQP4 was first observed by in situ hybridization in the rat brain, with expression of the messenger in the glia limitans, the ependymal lining system, the magnocellular hypothalamic nuclei, the cerebellum, the hippocampus, the neocortex and in the medial habenular nucleus (Jung et al. 1994; Venero et al. 1999). This regional distribution for AQP4 mRNA was confirmed by several immunohistochemistry studies (Nielsen et al. 1997; Badaut et al. 2000a, b). AQP4 protein is present on astrocyte endfeet in contact with blood vessels but also on astrocytic processes in contact with the synapses (Nielsen et al. 1997; Badaut et al. 2000a, b).

Electronic microscopy studies after cryofracture and immunogold techniques have shown that the highest density of AQP4 in astrocyte endfeet was observed in geometric structures named orthogonal arrays of proteins (OAPs; Verbavatz et al. 1997; Rash et al. 1998, 2004). Interestingly, the ratio between the expression of the long and the short AQP4 splice variant (AQP4m1 and AQP4m23) determines the size of the OAPs (Rash et al. 2004). The AQP4m23 isoform stabilizes the structure of the OAPs and an increase of its expression induces an increase in the size of the OAPs (Rash et al. 2004). The first functional role of the OAPs has been suggested to be an involvement with the astrocytic potassium-buffering (Grange-Messent et al. 1996). To date, functional consequences of the variation of the size of the OAPs are not known. Recently, another physiological role has been suggested for AQP4, in cell adhesion (Hiroaki et al. 2006). The involvement of an APQ in cell adhesion has been well described for epithelial cells of the lens, where AQP0 participates in the linkage of cells (Gonen et al. 2004). The high level of AQP4 protein in the hypothalamic glia lamellae should facilitate the adhesion between the astrocyte processes (Hiroaki et al. 2006). In this situation, AQP4 should not be involved in water diffusion but rather in cell adhesion between astrocytes and possibly to endothelial cells or muscle cells in the perivascular compartment. To support this idea, recent reports showed that the presence of AQP4 of the endfoot membrane is dependent on the presence of proteins in the basal lamina such as agrin, $\alpha$ dystroglycan and laminin (Guadagno and Moukhles 2004; Warth et al. 2004), suggesting an involvement in the ability of astrocytes to maintain the integrity of the blood-brain barrier. In the intracellular region, AQP4 is anchored to several proteins of the astrocytic cytoskeleton such as $\alpha 1$-syntrophin and dystrophin (Frigeri et al. 2001; Neely et al. 2001; Vajda et al. 2002; Amiry-Moghaddam et al. 2004). However, AQP4 is also observed outside the OAPs (Warth et al. 2004) raising the following questions: is there a water diffusion inside the OAPs? Is there a difference in the capacity of water diffusion inside or outside the OAPs? These questions are 
crucial because induction of AQP4 has been described in several brain diseases such as ischemia (Badaut et al. 2002).

Some new insights were obtained from two mice strains deficient in gene expression of dystrophin (mdx) and $\alpha 1$-syntrophin (a protein linked to dystrophin) which exhibit a marked decrease in AQP4 immunolabeling with swelling of astrocytic end-feet (Frigeri et al. 2001; Neely et al. 2001; Vajda et al. 2002; AmiryMoghaddam et al. 2004). In mdx and syntrophin knock out mice, astrocyte swelling may be due to impaired water elimination resulting from AQP4-disturbed organization at the plasma membrane. Another clue to the function of perivascular AQP4 was obtained by platelet-derived growth factor B (PDGF-B)-knockout mice, which showed abnormal vascular morphogenesis resulting in the absence of pericytes, and the presence of endothelial hyperplasia already at embryonic day 11.5 (Hellstrom et al. 2001). These knockout mice showed an increase in AQP4 concentration and swelling of astrocytes which may be a response to vascular abnormalities (Hellstrom et al. 2001). Similarly, a significant induction of AQP4 expression was observed on astrocyte endfeet in the ischemic hemisphere, one hour after stroke onset (de Castro Ribeiro et al. 2006). These results suggest that the increased AQP4 content of the perivascular space highlights the need for rapid water movements in this region. The pattern of distribution of aquaporins within the perivascular space might be related to the control of the perivascular volume, a function that may be crucial for maintenance of cerebral blood perfusion (Badaut et al. 2000b) and facilitation of the water clearance from perivascular space.

In the rodent brain, several regions show a strong AQP4 immunoreactive signal that may be due to the presence of the channel outside the perivascular space on astrocyte processes surrounding neuronal cells (Nielsen et al. 1997; Venero et al. 1999; Badaut et al. 2000b). The cellular distribution of AQP4 protein suggest that it may be involved in potassium homeostasis due to its co-distribution with KIR4.1 (Nagelhus et al. 2004). This hypothesis is also supported by functional results obtained from AQP4 KO mice showing that the delay of potassium re-uptake during electrical activity is increased and therefore these mice develop seizures more easily (Binder et al. 2006). The perivascular pool of AQP4 anchored by $\alpha$-syntrophin, seems to have an important role in this spatial potassium buffering because the delay of potassium re-uptake during electrical activity is also increased in $\alpha$-syntrophin KO mice (Amiry-Moghaddam et al. 2003b). Interestingly, observations in sclerotic tissues from patients with mesial temporal lobe epilepsy and hippocamplal sclerosis, suggest that the loss of perivascular AQP4 could be secondary due to the absence of brain specific dystrophin in these pathological tissues (Eid et al. 2005). This hypothesis suggests that the clearance of extracellular potassium can be compromised which then contributes to the accumulation of extracellular potassium in the brain of these patients. These results support the concept that AQP4 plays an important role in ionic homeostasis by facilitating water diffusion.

Potassium spatial buffering is facilitated by application of vasopressin (VP), an anti-diuretic hormone, which stimulates V1b receptors in acute cortical slices (Niermann et al. 2001). The increase in extracellular potassium clearance after VP application may be due an increase of AQP4 expression (Niermann et al. 2001). In contrast to brain, the effect of VP on the level of AQP4 and AQP2 expression is well 
described in the kidney (King et al. 2000). However, direct regulation of the level of AQP4 expression by VP has never been demonstrated in the central nervous system.

VP is synthesized in the magnocellular and parvocellular neurons of the hypothalamic nuclei, paraventricular and supraoptic nuclei and released into the blood stream in the neurohypophysis. These hypothalamic nuclei are known to be involved in the central osmoreception of variations of plasma osmotic pressure (Wells 1998). The high level of AQP4 and similar distribution have been observed in all osmosensitive brain areas such as the subfornical organ, the supraoptic nuclei, the paraventricular nucleus and accessory nuclei such as the circularis nucleus (Nielsen et al. 1997; Badaut et al. 2000a). In these brain regions, AQP4 is on plasma membranes facing both capillaries and magnocellular neurons (Nielsen et al. 1997; Badaut et al. 2000a). The specificity and intensity of AQP4 staining within hypothalamic magnocellular nuclei strongly suggest that water channels allow variations of plasma osmotic pressure to be transferred from blood to osmosensitive neurons. Furthermore, high blood vessel density is another common feature of these nuclei, which contribute to plasma osmolarity detection (Badaut et al. 2000a).

\section{AQP9 expression in brain and its functional roles}

Aquaglyceroporins facilitate the diffusion of water and several solutes such as glycerol, urea and monocarboxylate. The highest level of expression of AQP9 is in the liver (Tsukaguchi et al. 1998; Elkjaer et al. 2000) with polarization of the protein to the hepatocytic plasma membrane facing the sinusoids (Elkjaer et al. 2000; Nicchia et al. 2001; Nihei et al. 2001). The expression of AQP9 is also observed in the rodent brain (Badaut and Regli 2004). AQP9 mRNA was first detected in astrocytic cultures (Tsukaguchi et al. 1998) and confirmed by immunocytochemical studies in rodent brain (Badaut et al. 2001, 2004). To date, AQP9 expression has been observed in three cell types: glial cells, in particular tanycytes and astrocytes (Elkjaer et al. 2000; Badaut et al. 2001, 2004), endothelial cells of sub-pial vessels (Badaut et al. 2004) and neurons (Badaut et al. 2004; de Castro Ribeiro et al. 2006).

AQP9 expression was found predominantly in one subtype of neuronal cells, the catecholaminergic neurons, characterized by tyrosine hydroxylase expression in rat and mouse brains (Badaut et al. 2004; de Castro Ribeiro et al. 2006). In agreement with the regional distribution of AQP9 protein by immunohistochemistry, high levels of mRNA for AQP9 was detected in catecholaminergic nuclei confirming the presence of this protein in these nuclei (Badaut et al. 2004). Regarding these results, a sole role for AQP9 in water homeostasis should be revised, as to the best of our knowledge, catecholaminergic neurons are not directly implied in the regulation of systemic osmotic pressure, but are rather involved in energy balance (Grill and Kaplan 2002; Penicaud et al. 2002). This leads us to postulate that AQP9 could be involved in brain energy metabolism as a metabolite channel. In agreement with this hypothesis, the presence of AQP9 protein was recently demonstrated in mitochondria of astrocytes and dopaminergic neurons (Amiry-Moghaddam et al. 2005). Indeed, two isoforms of AQP9 are expressed in brain cells. The shortest isoform of AQP9 $(26 \mathrm{kDa})$ is observed in inner membrane of the mitochondria and the longest isoform $(30 \mathrm{kDa})$ is present in cell membrane (Amiry-Moghaddam et al. 2005). It is 
possible that the presence of AQP9 facilitates the diffusion of glycerol and monocarboxylates, which serve as energy substrates for neurons (McKenna et al. 1986; Magistretti et al. 1999; Nguyen et al. 2003). Interestingly, utilization of glycerol by neuronal cells in vitro was inhibited by mercury, suggesting transport by an AQP (Nguyen et al. 2003). In the "lactate shuttle" model, glucose is transformed by astrocytes into lactate and diffuses from astrocytes to neurons using the monocarboxylate transporters (Magistretti and Pellerin 1999). Therefore, presence of AQP9 in astrocytes suggests that it may facilitate the diffusion of lactate to neuronal cells in conjunction with the monocarboxylate transporters.

In addition, some catecholaminergic neurons are known to be sensitive to variations in glucose levels and recently, lactate and glycerol have been also shown to be potential activators of these neurons (Yang et al. 1999; Ainscow et al. 2002; Penicaud et al. 2002). These neurons are located in the same brain areas as AQP9positive neurons (Badaut and Regli 2004). Taken together, these results raise the hypothesis that neuronal AQP9 plays a role in energy balance as a glycerol-lactatechannel, but this awaits functional proof.

In liver, AQP9 expression has been shown to be regulated by the physiological feeding state mediated by the insulin response element (IRE) in the promoter of the gene (Kuriyama et al. 2002). High plasma insulin suppresses glycerol uptake into hepatocytes which participates in neoglucogenosis to replenish blood glucose concentrations. AQP9 is down regulated by high insulin concentration suggesting that AQP9 may play a key role in cellular energy balance as a glycerol channel in the liver (Kuriyama et al. 2002; Carbrey et al. 2003). In vivo, the expression of AQP9 is increased after fasting and returns to basal levels upon refeeding (Carbrey et al. 2003). AQP9 expression in liver is dramatically increased in models of diabetes induced by streptozotocin (STZ) injection (Carbrey et al. 2003) and in a mouse model of insulin resistance (Kuriyama et al. 2002). As mentioned above, energy balance is regulated by detection of glucose levels in the periphery as well as in the central nervous system (Levin et al. 1999). The feeding state, set by blood insulin concentration, also influences the glucose sensitive areas of the brain. Interestingly, AQP9-positive neurons are present in brain regions known to be glucose sensitive or implicated in the feeding state (Badaut et al. 2004); systemic insulin has been reported to cross the $\mathrm{BBB}$, and insulin receptors are expressed in catecholaminergic neurons (Kyriaki 2003; Unger et al. 1991 \#211). These data lead us to investigate whether brain AQP9 expression is regulated by insulin. The first in vitro results on brain stem slices containing catecholaminergic neurons showed a decrease in AQP9 protein levels $6 \mathrm{~h}$ after insulin application (Badaut et al. 2005). In vivo, the level of AQP9 was increased in liver and in the NTS of rats treated with STZ injections to induce diabetes (Badaut and Regli 2004; Badaut et al. 2005). Therefore, it would appear that brain AQP9 expression appears is regulated by insulin concentrations, supporting the hypothesis that AQP9 is involved in brain energy metabolism.

AQP9 was also observed in endothelial cells of the pial vessels, and in the intraparenchymal vessels (Badaut et al. 2004; Amiry-Moghaddam et al. 2005). interestingly in mice, AQP4 expression was observed by immunogold labeling in endothelial cells at their luminal, as well as abluminal membranes, but at much lower levels than observed on astrocytic endfeet (Amiry-Moghaddam et al. 2004). The blood-brain barrier (BBB) is known to be highly permeable to water (Oldendorf 包 Springer 
1970) and therefore, AQP4 and AQP9 may facilitate the water flow through endothelial cells. AQP9 could also participate in diffusion of monocarboxylate through the $\mathrm{BBB}$ which is mainly carried out by the monocarboxylate transporter, MCT1, highly expressed in endothelial cells (Gerhart et al. 1997; Pierre et al. 2000; Bergersen et al. 2002).

\section{AQP expression in brain disorders}

As mentioned previously, AQPs in rodent brain as water-channels are likely to play an important role in extracellular homeostasis, and thus may sustain normal neuronal activity (Badaut et al. 2002). A profound perturbation of the brain environment usually induces a regional cerebral edema, as observed in ischemia. Brain edema which leads to an expansion of brain volume, has a crucial impact on morbidity and mortality after stroke as it increases intracranial pressure, favours herniations, and contributes to additional ischemic injuries (Klatzo 1985). Despite its complexity, brain oedema has been defined as an increase in net brain water content which leads to an increase in tissue volume (Pappius 1974). The two major types of brain oedema, cytotoxic and vasogenic oedema, both occur after brain ischemia. Cytotoxic oedema is characterized by intracellular water accumulation involving both astrocytes and neurons and depending mainly on the perturbation of ionic gradients (Kimelberg 2004). Vasogenic oedema is characterized by a protein rich exudate derived from plasma, as a result of an increased permeability of the capillary endothelial cells to albumin and other plasma proteins (Unterberg et al. 2004).

As mentioned previously, three AQPs could be involved in water movements occurring during formation and resolution of cerebral edema after ischemia. Astrocytic AQP1 expression is induced in human brain tissues after subarachnoid hemorrhage (SAH; Badaut et al. 2003) but modification in expression has not yet been described in rodent models of human brain disorders (de Castro Ribeiro et al. 2006). The level of AQP4 expression is regulated in several brain disorders, such as trauma (Ke et al. 2001; Kiening et al. 2002; Sun et al. 2003), ischemia (Taniguchi et al. 2000; Amiry-Moghaddam et al. 2003a; Meng et al. 2004; de Castro Ribeiro et al. 2006) and human SAH (Badaut et al. 2003). To better understand the roles of AQP4 and AQP9 in edema, the expression profiles were recently characterized at various time points after transient cerebral ischemia in mice. Two peaks of AQP4 expression were observed $1 \mathrm{~h}$ and $48 \mathrm{~h}$ after stroke, coinciding with the two peaks of maximal hemispheric swelling (de Castro Ribeiro et al. 2006). This temporal expression of AQP4 differs with the result from brain trauma where there is an initial decrease in AQP4 levels within 48 hours, followed by an increase (Ke et al. 2001, 2002; Kiening et al. 2002). The difference in expression between the two models suggests that the role of AQP4 in edema formation and resolution is complex. In contrast to AQP4, AQP9 showed a significant induction at $24 \mathrm{~h}$, that increased gradually with time, without correlation to swelling (de Castro Ribeiro et al. 2006), suggesting that AQP4 but not AQP9 plays a role in edema formation after transient cerebral ischemia in the mouse. Interestingly, AQP4 expression is rapidly regulated with a major induction at $1 \mathrm{~h}$ after stroke onset on astrocyte endfeet, suggesting that we need to consider early time points after brain disorder onset (de Castro Ribeiro et al. 
2006). To date, functional consequences of the early AQP4 induction on astrocyte endfeet have not yet been determined. It is possible that the presence of AQP4 in cytotoxic edema formation is deleterious (Manley et al. 2000). Indeed, edema is lower in AQP4-KO mice compared to wild type after permanent ischemia and acute water intoxication (Manley et al. 2000). On the other hand, AQP4 was shown to be important in water clearance in vasogenic edema (Papadopoulos et al. 2004). Perhaps, AQP4 plays a dual role in edema evolution. However, a treatment with sulforaphane which enhances AQP4 expression, induces a decrease in edema following traumatic brain injury (Zhao et al. 2005), suggesting that induction of AQP4 expression could facilitate the water clearance to decrease edema.

As mentioned previously, the role of AQP4 in brain disorders is complex. Indeed, AQP4 has been recently shown to be involved in glial scar formation due to its involvement in astrocyte migration towards the lesion (Saadoun et al. 2005). This new role for AQP4 illustrates well that one AQP could be involved in several cellular functions in one organ and in one cell type, depending on the environment.

AQP9 protein is up-regulated on reactive astrocytes in the border of the infarct after transient middle cerebral artery occlusion in mice (Badaut et al. 2001; de Castro Ribeiro et al. 2006). This up-regulation of AQP9 in reactive astrocytes was also observed in the border zone not influenced by the middle cerebral artery territory (de Castro Ribeiro et al. 2006). AQP9 permeability to water, glycerol and lactate may be important under pathological conditions such as brain ischemia (Bertrand et al. 1992; Schulz et al. 2000; Frykholm et al. 2001; Kuo et al. 2003) and interestingly, AQP9 permeability to lactate increases fourfold when the $\mathrm{pH}$ decreases to 5.5 (Tsukaguchi et al. 1998). Lactic acidosis during ischemia may increase the permeability of AQP9 and enable uptake of excess lactate by astrocytes. In this way AQP9 could favor lactate and glycerol clearing from the extracellular space during ischemia. Lactate and glycerol could then be used as energetic substrates, lactate, for example, has been shown to facilitate the recovery of neurons after ischemic insults (Schurr 2002).

To date, molecular pathways involved in AQP4 and 9 regulation have not yet been studied in vivo. However several groups have investigated the regulation of AQP expression in primary astrocyte cultures. Several pathways leading to regulation of $\mathrm{AQP}$ expression were identified including protein kinase A (PKA) and C (PKC; Yamamoto et al. 2001, 2002). Stimulation of the PKC pathway is known to reduce AQP4 mRNA expression in astrocyte cultures (Nakahama et al. 1999) and furthermore, PKC phosphorylation at consensus sites in the AQP4 protein acts to reduce water influx through the channel (Han et al. 1998; Vajda et al. 2000). AQP9 mRNA and protein were also down-regulated by stimulation of the PKC pathway which did not require de novo protein synthesis (Yamamoto et al. 2001). Despite the presence of consensus sites for phosphorylation by PKC in the AQP9 protein, direct regulation of this channel by phosphorylation has not yet been observed (Yamamoto et al. 2001). Activation of PKA by dibutirylcAMP induces an increase in AQP9 mRNA and protein expression in contrast to AQP4 in astrocytic cultures (Yamamoto et al. 2002). Recently, P38 MAP-kinase was shown to be involved in an increase of AQP4 and AQP9 expression after osmotic stress (Arima et al. 2003). MAP-kinase pathways is activated in ischemia and then could be also involved in the increase of astrocytic AQP9 expression at the border of the ischemic infarct (Badaut et al. 2001; de Castro Ribeiro et al. 2006).

黑 Springer 


\section{Conclusion}

Regarding to recent advances, a unique role of AQP in brain water homeostasis needs to be reconsidered. AQP4 is involved in ionic homeostasis of the brain by facilitation of water diffusion through the cell membrane. An absence of AQP4 in astrocyte endfeet modifies the clearance of potassium during neuronal activity and leads to an increase in the susceptibility of animals to seizure. In pathological conditions, expression of AQP4 is rapidly increased and correlated with edema formation. However, the exact role of AQP4 in edema formation is still debated. Furthermore, the induction of AQP4 in reactive astrocytes after traumatic brain injury has been recently associated with astrocyte migrations towards the glia scar.

AQP9 is expressed in astrocytes and catecholaminergic neurons and may be involved in brain energy metabolism by facilitating the diffusion of solutes such as glycerol and monocarboxylates. Furthermore, the level of AQP9 expression is dependent on the concentration of insulin, supporting the idea that AQP9 is involved in brain energy metabolism. In ischemia, the AQP9 expression is induced over time and could participate in the reuptake of excess glycerol and lactate after stroke onset. Recent reports on AQP9 suggest that the AQP is not only an aqueduct but a metabolite pipeline.

Acknowledgements The authors wish thank Dr M. Price for critical comments on the manuscript. This study was supported by grants from the Swiss Science Foundation (FN 3100AO-108001); Fondazione Per Lo Studio Delle Malattie Neurodegenerative Delle Persone Adulte e Dell' Anziano", from Lugano, Switzerland; SwissHeart foundation.

\section{References}

Ainscow EK, Mirshamsi S, Tang T, Ashford ML, Rutter GA (2002) Dynamic imaging of free cytosolic ATP concentration during fuel sensing by rat hypothalamic neurones: evidence for ATP-independent control of ATP-sensitive K(+) channels. J Physiol 544:429-445

Amiry-Moghaddam M, Ottersen OP (2003) The molecular basis of water transport in the brain. Nat Rev Neurosci 4:991-1001

Amiry-Moghaddam M, Otsuka T, Hurn PD, Traystman RJ, Haug FM, Froehner SC, Adams ME, Neely JD, Agre P, Ottersen OP, Bhardwaj A (2003a) An alpha-syntrophin-dependent pool of AQP4 in astroglial end-feet confers bidirectional water flow between blood and brain. Proc Natl Acad Sci U S A 100:2106-2111

Amiry-Moghaddam M, Williamson A, Palomba M, Eid T, de Lanerolle NC, Nagelhus EA, Adams ME, Froehner SC, Agre P, Ottersen OP (2003b) Delayed K+clearance associated with aquaporin-4 mislocalization: phenotypic defects in brains of alpha-syntrophin-null mice. Proc Natl Acad Sci U S A 100:13615-13620

Amiry-Moghaddam M, Xue R, Haug FM, Neely JD, Bhardwaj A, Agre P, Adams ME, Froehner SC, Mori S, Ottersen OP (2004) Alpha-syntrophin deletion removes the perivascular but not endothelial pool of aquaporin-4 at the blood-brain barrier and delays the development of brain edema in an experimental model of acute hyponatremia. FASEB J 18:542-544

Amiry-Moghaddam M, Lindland H, Zelenin S, Roberg BA, Gundersen BB, Petersen P, Rinvik E, Torgner IA, Ottersen OP (2005) Brain mitochondria contain aquaporin water channels: evidence for the expression of a short AQP9 isoform in the inner mitochondrial membrane. FASEB J 19:1459-1467

Arima H, Yamamoto N, Sobue K, Umenishi F, Tada T, Katsuya H, Asai K (2003) Hyperosmolar mannitol stimulates expression of aquaporin 4 and 9 through a p38 mitogen activated protein kinase-dependent pathway in rat astrocytes. J Biol Chem 27:27

Badaut J, Regli L (2004) Distribution and possible roles of aquaporin 9 in the brain. Neuroscience 129:969-979 
Badaut J, Nehlig A, Verbavatz J, Stoeckel M, Freund-Mercier MJ, Lasbennes F (2000a) Hypervascularization in the magnocellular nuclei of the rat hypothalamus: relationship with the distribution of aquaporin-4 and markers of energy metabolism. J Neuroendocrinol 12:960-969

Badaut J, Verbavatz JM, Freund-Mercier MJ, Lasbennes F (2000b) Presence of aquaporin-4 and muscarinic receptors in astrocytes and ependymal cells in rat brain: a clue to a common function. Neurosci Lett 292:75-78

Badaut J, Hirt L, Granziera C, Bogousslavsky J, Magistretti PJ, Regli L (2001) Astrocyte-specific expression of aquaporin-9 in mouse brain is increased after transient focal cerebral ischemia. $\mathrm{J}$ Cereb Blood Flow Metab 21:477-482

Badaut J, Lasbennes F, Magistretti PJ, Regli L (2002) Aquaporins in brain: distribution, physiology, and pathophysiology. J Cereb Blood Flow Metab 22:367-378

Badaut J, Brunet JF, Grollimund L, Hamou MF, Magistretti PJ, Villemure JG, Regli L (2003) Aquaporin 1 and aquaporin 4 expression in human brain after subarachnoid hemorrhage and in peritumoral tissue. Acta Neurochir Suppl 86:495-498

Badaut J, Petit JM, Brunet JF, Magistretti PJ, Charriaut-Marlangue C, Regli L (2004) Distribution of Aquaporin 9 in the adult rat brain: preferential expression in catecholaminergic neurons and in glial cells. Neuroscience 128:27-38

Badaut J, Petit JM, Brunet JF, Magistretti P, Regli F (2005) Brain aquaporin 9 (AQP9) regulation by systemic insulin in rat. J Cereb Blood Flow Metab 25:246

Bergersen L, Rafiki A, Ottersen OP (2002) Immunogold cytochemistry identifies specialized membrane domains for monocarboxylate transport in the central nervous system. Neurochem Res 27:89-96

Bertrand N, Ishii H, Spatz M (1992) Regional and temporal glycerol changes induced by forebrain ischemia in gerbils. Neurosci Lett 148:81-84

Binder DK, Yao X, Zador Z, Sick TJ, Verkman AS, Manley GT (2006) Increased seizure duration and slowed potassium kinetics in mice lacking aquaporin-4 water channels. Glia 53:631-636

Brown PD, Davies SL, Speake T, Millar ID (2004) Molecular mechanisms of cerebrospinal fluid production. Neuroscience 129:955-968

Carbrey JM, Gorelick-Feldman DA, Kozono D, Praetorius J, Nielsen S, Agre P (2003) Aquaglyceroporin AQP9: solute permeation and metabolic control of expression in liver. Proc Natl Acad Sci U S A 100:2945-2950

Cooper GJ, Zhou Y, Bouyer P, Grichtchenko II, Boron WF (2002) Transport of volatile solutes through AQP1. J Physiol 542:17-29

de Castro Ribeiro M, Hirt L, Bogousslavsky J, Regli L, Badaut J (2006) Time course of aquaporin expression after transient focal cerebral ischemia in mice. J Neurosci Res 83:1231-1240

Dolman D, Drndarski S, Abbott NJ, Rattray M (2005) Induction of aquaporin 1 but not aquaporin 4 messenger RNA in rat primary brain microvessel endothelial cells in culture. J Neurochem 93:825-833

Eid T, Lee TS, Thomas MJ, Amiry-Moghaddam M, Bjornsen LP, Spencer DD, Agre P, Ottersen OP, de Lanerolle NC (2005) Loss of perivascular aquaporin 4 may underlie deficient water and $\mathrm{K}+$ homeostasis in the human epileptogenic hippocampus. Proc Natl Acad Sci U S A 102:1193-1198

Elkjaer M, Vajda Z, Nejsum LN, Kwon T, Jensen UB, Amiry-Moghaddam M, Frokiaer J, Nielsen S (2000) Immunolocalization of AQP9 in liver, epididymis, testis, spleen, and brain. Biochem Biophys Res Commun 276:1118-1128

Frigeri A, Nicchia GP, Nico B, Quondamatteo F, Herken R, Roncali L, Svelto M (2001) Aquaporin-4 deficiency in skeletal muscle and brain of dystrophic mdx mice. FASEB J 15:90-98

Frykholm P, Hillered L, Langstrom B, Persson L, Valtysson J, Watanabe Y, Enblad P (2001) Increase of interstitial glycerol reflects the degree of ischaemic brain damage: a PET and microdialysis study in a middle cerebral artery occlusion-reperfusion primate model. J Neurol Neurosurg Psychiatry 71:455-461

Gerhart DZ, Enerson BE, Zhdankina OY, Leino RL, Drewes LR (1997) Expression of monocarboxylate transporter MCT1 by brain endothelium and glia in adult and suckling rats. Am J Physiol 273:E207-E213

Gonen T, Sliz P, Kistler J, Cheng Y, Walz T (2004) Aquaporin-0 membrane junctions reveal the structure of a closed water pore. Nature 429:193-197

Grange-Messent V, Raison D, Bouchaud C (1996) Compared effects of extracellular K + ions and soman, a neurotoxic, on cerebral astrocyte morphology. An in vitro study. J Submicrosc Cytol Pathol 28:151-159

Grill HJ, Kaplan JM (2002) The neuroanatomical axis for control of energy balance. Front Neuroendocrinol 23:2-40

Guadagno E, Moukhles H (2004) Laminin-induced aggregation of the inwardly rectifying potassium channel, Kir4.1, and the water-permeable channel, AQP4, via a dystroglycan-containing complex in astrocytes. Glia 47:138-149

Han Z, Wax MB, Patil RV (1998) Regulation of aquaporin-4 water channels by phorbol ester-dependent protein phosphorylation. J Biol Chem 273:6001-6004 
Hellstrom M, Gerhardt H, Kalen M, Li X, Eriksson U, Wolburg H, Betsholtz C (2001) Lack of pericytes leads to endothelial hyperplasia and abnormal vascular morphogenesis. J Cell Biol 153:543-553

Hiroaki Y, Tani K, Kamegawa A, Gyobu N, Nishikawa K, Suzuki H, Walz T, Sasaki S, Mitsuoka K, Kimura K (2006) Implications of the aquaporin-4 structure on array formation and cell adhesion. J Mol Biol 355:628-639

Ishibashi K, Kuwahara M, Gu Y, Tanaka Y, Marumo F, Sasaki S (1998) Cloning and functional expression of a new aquaporin (AQP9) abundantly expressed in the peripheral leukocytes permeable to water and urea, but not to glycerol. Biochem Biophys Res Commun 244:268-274

Jung JS, Bhat RV, Preston GM, Guggino WB, Baraban JM, Agre P (1994) Molecular characterization of an aquaporin cDNA from brain: candidate osmoreceptor and regulator of water balance. Proc Natl Acad Sci U S A 91:13052-13056

Ke C, Poon WS, Ng HK, Pang JC, Chan Y (2001) Heterogeneous responses of aquaporin-4 in oedema formation in a replicated severe traumatic brain injury model in rats. Neurosci Lett 301:21-24

Ke C, Poon WS, Ng HK, Lai FM, Tang NL, Pang JC (2002) Impact of experimental acute hyponatremia on severe traumatic brain injury in rats: influences on injuries, permeability of blood-brain barrier, ultrastructural features, and aquaporin-4 expression. Exp Neurol 178:194-206

Kiening KL, van Landeghem FK, Schreiber S, Thomale UW, von Deimling A, Unterberg AW, Stover JF (2002) Decreased hemispheric aquaporin-4 is linked to evolving brain edema following controlled cortical impact injury in rats. Neurosci Lett 324:105-108

Kimelberg HK (2004) Water homeostasis in the brain: Basic concepts. Neuroscience 129:851-860

King LS, Yasui M, Agre P (2000) Aquaporins in health and disease. Mol Med Today 6:60-65

Klatzo I (1985) Brain oedema following brain ischaemia and the influence of therapy. Br J Anaesth 57:18-22

Ko SB, Uchida S, Naruse S, Kuwahara M, Ishibashi K, Marumo F, Hayakawa T, Sasaki S (1999) Cloning and functional expression of rAOP9L a new member of aquaporin family from rat liver. Biochem Mol Biol Int 47:309-318

Kuo JR, Lin CL, Chio CC, Wang JJ, Lin MT (2003) Effects of hypertonic (3\%) saline in rats with circulatory shock and cerebral ischemia after heatstroke. Intensive Care Med 29:1567-1573

Kuriyama H, Shimomura I, Kishida K, Kondo H, Furuyama N, Nishizawa H, Maeda N, Matsuda M, Nagaretani H, Kihara S, Nakamura T, Tochino Y, Funahashi T, Matsuzawa Y (2002) Coordinated regulation of fat-specific and liver-specific glycerol channels, aquaporin adipose and aquaporin 9 . Diabetes 51:2915-2921

Kyriaki G (2003) Brain insulin: regulation, mechanisms of action and functions. Cell Mol Neurobiol 23:1-25

Levin BE, Dunn-Meynell AA, Routh VH (1999) Brain glucose sensing and body energy homeostasis: role in obesity and diabetes. Am J Physiol 276:R1223-R1231

Liu Z, Shen J, Carbrey JM, Mukhopadhyay R, Agre P, Rosen BP (2002) Arsenite transport by mammalian aquaglyceroporins AQP7 and AQP9. Proc Natl Acad Sci U S A 99:6053-6058

Magistretti PJ, Pellerin L (1999) Cellular mechanisms of brain energy metabolism and their relevance to functional brain imaging. Philos Trans R Soc Lond B Biol Sci 354:1155-1163

Magistretti PJ, Pellerin L, Rothman DL, Shulman RG (1999) Energy on demand. Science 283:496-497

Manley GT, Fujimura M, Ma T, Noshita N, Filiz F, Bollen AW, Chan P, Verkman AS (2000) Aquaporin-4 deletion in mice reduces brain edema after acute water intoxication and ischemic stroke. Nat Med 6:159-163

McKenna MC, Bezold LI, Kimatian SJ, Tildon JT, Fife MM (1986) Competition of glycerol with other oxidizable substrates in rat brain. Biochem $\mathrm{J}$ 237:47-51

Meng S, Qiao M, Lin L, Del Bigio MR, Tomanek B, Tuor UI (2004) Correspondence of AQP4 expression and hypoxic-ischaemic brain oedema monitored by magnetic resonance imaging in the immature and juvenile rat. Eur J Neurosci 19:2261-2269

Nagelhus EA, Mathiisen TM, Ottersen OP (2004) Aquaporin-4 in the central nervous system: Cellular and subcellular distribution and coexpression with KIR4.1. Neuroscience 129:905-913

Nakahama K, Nagano M, Fujioka A, Shinoda K, Sasaki H (1999) Effect of TPA on aquaporin 4 mRNA expression in cultured rat astrocytes. Glia 25:240-246

Neely JD, Amiry-Moghaddam M, Ottersen OP, Froehner SC, Agre P, Adams ME (2001) Syntrophindependent expression and localization of Aquaporin-4 water channel protein. Proc Natl Acad Sci U S A 98:14108-14113

Nguyen NH, Brathe A, Hassel B (2003) Neuronal uptake and metabolism of glycerol and the neuronal expression of mitochondrial glycerol-3-phosphate dehydrogenase. J Neurochem 85:831-842

Nicchia GP, Frigeri A, Nico B, Ribatti D, Svelto M (2001) Tissue distribution and membrane localization of aquaporin-9 water channel: evidence for sex-linked differences in liver. J Histochem Cytochem 49:1547-1556 
Nielsen S, Smith BL, Christensen EI, Agre P (1993) Distribution of the aquaporin CHIP in secretory and resorptive epithelia and capillary endothelia. Proc Natl Acad Sci U S A 90:7275-7279

Nielsen S, Nagelhus EA, Amiry-Moghaddam M, Bourque C, Agre P, Ottersen OP (1997) Specialized membrane domains for water transport in glial cells: high-resolution immunogold cytochemistry of aquaporin-4 in rat brain. J Neurosci 17:171-180

Niermann H, Amiry-Moghaddam M, Holthoff K, Witte OW, Ottersen OP (2001) A novel role of vasopressin in the brain: modulation of activity-dependent water flux in the neocortex. J Neurosci 21:3045-3051

Nihei K, Koyama Y, Tani T, Yaoita E, Ohshiro K, Adhikary LP, Kurosaki I, Shirai Y, Hatakeyama K, Yamamoto T (2001) Immunolocalization of aquaporin-9 in rat hepatocytes and Leydig cells. Arch Histol Cytol 64:81-88

Oldendorf WH (1970) Measurement of brain uptake of radiolabeled substances using a tritiated water internal standard. Brain Res 24:372-376

Papadopoulos MC, Manley GT, Krishna S, Verkman AS (2004) Aquaporin-4 facilitates reabsorption of excess fluid in vasogenic brain edema. FASEB J 18:1291-1293

Pappius HM (1974) Part I: tumors of the brain and skull. In: Vinken PJ, Bruyn GW (eds) Handbook of clinical neurology, vol. 16. North Holland Publishing Company, New York, pp 167-185

Penicaud L, Leloup C, Lorsignol A, Alquier T, Guillod E (2002) Brain glucose sensing mechanism and glucose homeostasis. Curr Opin Clin Nutr Metab Care 5:539-543

Pierre K, Pellerin L, Debernardi R, Riederer BM, Magistretti PJ (2000) Cell-specific localization of monocarboxylate transporters, MCT1 and MCT2, in the adult mouse brain revealed by double immunohistochemical labeling and confocal microscopy. Neuroscience 100:617-627

Preston GM, Carroll TP, Guggino WB, Agre P (1992) Appearance of water channels in Xenopus oocytes expressing red cell CHIP28 protein. Science 256:385-387

Rash JE, Yasumura T, Hudson CS, Agre P, Nielsen S (1998) Direct immunogold labeling of aquaporin-4 in square arrays of astrocyte and ependymocyte plasma membranes in rat brain and spinal cord. Proc Natl Acad Sci U S A 95:11981-11986

Rash JE, Davidson KGV, Yasumura T, Furman CS (2004) Freeze-fracture and immunogold analysis of aquaporin-4 (AQP4) square arrays, with models of AQP4 lattice assembly. Neuroscience 129:915-934

Saadoun S, Papadopoulos MC, Watanabe H, Yan D, Manley GT, Verkman AS (2005) Involvement of aquaporin-4 in astroglial cell migration and glial scar formation. J Cell Sci 118:5691-5698

Santoni V, Gerbeau P, Javot H, Maurel C (2000) The high diversity of aquaporins reveals novel facets of plant membrane functions. Curr Opin Plant Biol 3:476-481

Schulz MK, Wang LP, Tange M, Bjerre P (2000) Cerebral microdialysis monitoring: determination of normal and ischemic cerebral metabolisms in patients with aneurysmal subarachnoid hemorrhage. $\mathrm{J}$ Neurosurg 93:808-814

Schurr A (2002) Lactate, glucose and energy metabolism in the ischemic brain (Review). Int J Mol Med 10:131-136

Sun MC, Honey CR, Berk C, Wong NL, Tsui JK (2003) Regulation of aquaporin-4 in a traumatic brain injury model in rats. J Neurosurg 98:565-569

Taniguchi M, Yamashita T, Kumura E, Tamatani M, Kobayashi A, Yokawa T, Maruno M, Kato A, Ohnishi T, Kohmura E, Tohyama M, Yoshimine T (2000) Induction of aquaporin-4 water channel mRNA after focal cerebral ischemia in rat. Brain Res Mol Brain Res 78:131-137

Tsukaguchi H, Shayakul C, Berger UV, Mackenzie B, Devidas S, Guggino WB, van Hoek AN, Hediger MA (1998) Molecular characterization of a broad selectivity neutral solute channel. J Biol Chem $273: 24737-24743$

Tsukaguchi H, Weremowicz S, Morton CC, Hediger MA (1999) Functional and molecular characterization of the human neutral solute channel aquaporin-9. Am J Physiol 277:F685-F696

Unger JW, Livingston JN, Moss AM (1991) Insulin receptors in the central nervous system: localization, signalling mechanisms and functional aspects. Prog Neurobiol 36:343-362

Unterberg AW, Stover J, Kress B, Kiening KL (2004) Edema and brain trauma. Neuroscience 129: $1019-1027$

Vajda Z, Promeneur D, Doczi T, Sulyok E, Frokiaer J, Ottersen OP, Nielsen S (2000) Increased aquaporin4 immunoreactivity in rat brain in response to systemic hyponatremia. Biochem Biophys Res Commun 270:495-503

Vajda Z, Pedersen M, Fuchtbauer EM, Wertz K, Stodkilde-Jorgensen H, Sulyok E, Doczi T, Neely JD, Agre P, Frokiaer J, Nielsen S (2002) Delayed onset of brain edema and mislocalization of aquaporin-4 in dystrophin-null transgenic mice. Proc Natl Acad Sci U S A 99:13131-13136 
Venero JL, Vizuete ML, Ilundain AA, Machado A, Echevarria M, Cano J (1999) Detailed localization of aquaporin-4 messenger RNA in the CNS: preferential expression in periventricular organs. Neuroscience 94:239-250

Verbavatz JM, Ma T, Gobin R, Verkman AS (1997) Absence of orthogonal arrays in kidney, brain and muscle from transgenic knockout mice lacking water channel aquaporin-4. J Cell Sci 110:2855-2860

Warth A, Kroger S, Wolburg H (2004) Redistribution of aquaporin-4 in human glioblastoma correlates with loss of agrin immunoreactivity from brain capillary basal laminae. Acta Neuropathol (Berl) 107:311-318

Wells T (1998) Vesicular osmometers, vasopression secretion and aquaporin-4: a new mechanism for osmoreception. Mol Cell Endocrinol 136:103-107

Yamamoto N, Sobue K, Miyachi T, Inagaki M, Miura Y, Katsuya H, Asai K (2001) Differential regulation of aquaporin expression in astrocytes by protein kinase C. Brain Res Mol Brain Res 95:110-116

Yamamoto N, Sobue K, Fujita M, Katsuya H, Asai K (2002) Differential regulation of aquaporin-5 and -9 expression in astrocytes by protein kinase A. Brain Res Mol Brain Res 104:96-102

Yang XJ, Kow LM, Funabashi T, Mobbs CV (1999) Hypothalamic glucose sensor: similarities to and differences from pancreatic beta-cell mechanisms. Diabetes 48:1763-1772

Zhao J, Moore AN, Clifton GL, Dash PK (2005) Sulforaphane enhances aquaporin-4 expression and decreases cerebral edema following traumatic brain injury. J Neurosci Res 82:499-506 